Nervenarzt 2014 · 85:399-400

DOI 10.1007/s00115-013-3907-4

Online publiziert: 23. März 2014

๑) Springer-Verlag Berlin Heidelberg 2014

\section{H.-P-Kapfhammer ${ }^{1} \cdot$ R. Dodel ${ }^{2}$}

${ }^{1}$ Universitätsklinik für Psychiatrie, Medizinische Universität Graz

${ }^{2}$ Klinik und Poliklinik für Neurologie, Philipps-Universität Marburg, Universitätsklinikum Gießen und Marburg

\section{Multimorbidität}

\section{Eine zentrale Herausforderung der modernen Medizin}

Die Fortschritte der modernen Medizin im Kontext prinzipiell verbesserter Lebensbedingungen in den hoch industrialisierten Gesellschaften haben entscheidend dazu beigetragen, dass die mittlere Lebenserwartung signifikant gestiegen ist und sich der allgemeine Gesundheitsstatus bedeutsam verbessert hat. Mit dem Bevölkerungswandel und dem zunehmenden Anteil älterer Personen wächst als Kehrmedaille dieser Entwicklung aber epidemiologisch auch die Wahrscheinlichkeit, im Laufe des individuellen Lebens sukzessiv und/oder simultan mehrere ernsthafte Erkrankungen zu erwerben. Das Thema Komorbidität bzw. Multimorbidität, das auf allen Ebenen der medizinischen Versorgung, aber auch der gesundheitspolitischen Organisation eine ernstzunehmende Herausforderung darstellt, nimmt mittlerweile eine zentrale Rolle in modernen Gesundheitssystemen ein.

In einem einführenden Beitrag widmet sich R. Dodel der Klärung der Konzepte von Komorbidität und Multimorbidität. In seiner epidemiologischen Analyse der Literatur verdeutlicht er, dass Multimorbidität kein exklusives Problem des höheren Lebensalters darstellt, sondern auch in jüngeren Lebensabschnitten von großer Relevanz ist. Er weist auf eine bedeutsame geschlechtsdifferenzielle Dimension und auf grundlegende sozioökonomische Einflussvariablen hin. Er unterstreicht, dass in der Perspektivität von Multimorbidität nicht nur ein additives „Mehr“ an definierten Einzelerkrankungen bei einem Einzelpatienten gleichzeitig zu berücksichtigen und zusätzliche Therapien arithmetisch miteinander $\mathrm{zu}$ kombinieren sind. Multimorbidität verlangt als herausragendes versorgungspolitisches Thema die qualitativ neuartige Besinnung auf ein biopsychosoziales Krankheitsverständnis, das neben den Anforderungen an eine evidenzbasierte ärztliche Praxis und den Mehrkosten für das Gesundheitssystem konsequent auch die vielfältigen Auswirkungen von Mehrfacherkrankungen und Komplexbehandlungen auf das betroffene Individuum in seinem psychologischen Erleben und seiner psychosozialen Lebenswelt reflektiert.

S. Lorenzl diskutiert das Thema Multimorbidität am Beispiel von Spätstadien neurologischer Erkrankungen unter den speziellen Behandlungs- und Betreuungsbedingungen einer Palliativstation. In diesem besonderen Versorgungskontext am Lebensende bedeutet Multimorbidität auch und insbesondere fast regelhaft Demenz, Delir, epileptische Anfälle und iatrogen durch eine PEG-Ernährung herbeigeführte Symptome. Das systematische Überdenken von Polypharmazie und künstlicher Ernährung, das kritische Abwägen von Vor- und Nachteilen einzelner Therapien kann sich in diesem Abschnitt nicht am alleinbestimmenden Ziel einer abstrakten Lebensverlängerung orientieren, sondern muss in einer komplexen ärztlich-ethischen Erörterung stimmige Entscheidungen ermöglichen, die systematisch die Lebensqualität des einzelnen Patienten berücksichtigen. Diese speziell geforderte ärztliche Sensibilität bedeutet häufig auch das überlegte Absetzen von Behandlungen und Maßnahmen, die in diesem Stadium mit unzumutbaren Belastungen und Beeinträchtigungen für den einzelnen Patienten einhergehen und den natürlichen Sterbeprozess behindern. Der Autor betont, wie grundlegend für ein gelingendes patientenzentriertes Vorgehen auch das fortlaufende Gespräch mit den Angehörigen und ihre Miteinbeziehung in wesentliche Entscheidungen ist.

y) Pharmakologische
Informationssysteme können
ärztliche Entscheidungen
kooperativ unterstützen

Polypharmazie ist auf der Behandlungsebene ein von Multimorbidität untrennbares Korrelat. Angesichts der prinzipiell häufigen Nebenwirkungen unterschiedlicher medikamentöser Therapien und oft unübersichtlicher Interaktionen bei pharmakologischen Mehrfachkombinationen besteht bei Ärzten in der konkreten medikamentösen Planung begreiflicherweise große Unsicherheit. Diese steigert sich angesichts potenziell schwerwiegender Interaktionen und einer auch justiziablen ärztlichen Verantwortung nicht selten zu einer unproduktiven, daher eine im Einzelfall geforderte Therapiegestaltung behindernden Ängstlichkeit. E. Haen gibt einen hilfreichen Überblick über die grundlegenden pharmakokinetischen und pharmakodynamischen Prinzipien von Fremdstoffinteraktionen. Er diskutiert den Stellenwert des therapeutischen Drug-Monitorings und ermutigt zum Gebrauch pharmakologischer Informationssysteme, die den konkreten ärztlichen Entscheidungs- und Handlungsalltag kooperativ und kollegial unterstüt- 
zen können. Er rät hingegen von automatisierten, den individuellen Fall in seiner multimorbiden Ausgangssituation und multimodalen Therapieanforderung eben nicht berücksichtigenden und reflektierenden Auskunftsdateien als Entscheidungsgrundlage für eine gelingende rationale und rationelle Pharmakotherapie ab.

Das Auftreten eines Delirs nicht als zwar mögliche, aber allenfalls als nur passagere Komplikation eines multimorbiden Krankheitsprozesses fehleinzustufen, sondern vielmehr als ein alarmierendes neuropsychiatrisches Syndrom mit grundlegender Relevanz für die künftige somatische und mentale Morbidität und auch Mortalität zu kennzeichnen, ist die Absicht des Beitrags von K. Hüfner und B. Sperner-Unterweger. Sie skizzieren das Delir in seiner psychopathologischen Vielfalt als einen psychiatrischen Notfall, der regelhaft mit einer grundlegenden Erschütterung und Suspendierung eines geordneten Selbstverständnisses und personalen Handelns, mit möglicher Selbstund Fremdgefährdung und hohen Irritation des familiären und sozialen Umfelds einhergeht. Sie berichten epidemiologische Daten zur Delirhäufigkeit bei grundlegenden neurologischen Erkrankungen. In einer diagnostischen Perspektive ist es einerseits entscheidend wichtige allgemeine Risikovariablen zu erfassen, andererseits die speziellen Interaktionen multipler Krankheitsprozesse und aktueller somatisch-medizinischer Therapien in ihren delirfördernden Auswirkungen zu identifizieren und nach Möglichkeit zu korrigieren. Die Bedeutung einer raschen Symptomkontrolle mittels differenzieller antipsychotischer Medikation wird ebenso herausgestellt wie auch grundlegende Prinzipien nichtmedikamentöser „Multicomponent-Interventionen “ in der ärztlichen und pflegerischen Betreuung deliranter Patienten betont werden.

H.P. Kapfhammer widmet sich dem Thema der psychiatrischen Komorbidität bei neurologischen Erkrankungen. Er schildert am Beispiel der Koexistenz von depressiven und Angststörungen bei Patienten nach Schlaganfall und mit Parkinson-Syndromen zunächst die vielfältigen nachteiligen Konsequenzen in der aktuellen Erkrankung und im weiteren Verlauf. Diese implizieren ein höheres subjekti- ves Leiden, ein reduziertes Coping, mögliche negative Interferenzen mit somatischen Therapien und Rehabilitationen, eine stärkere Beeinträchtigung der Lebensqualität sowie höhere Grade an psychosozialer Behinderung. Sie schließen oft auch ein höheres somatisches Morbiditäts- und Mortalitätsrisiko ein. Das Verständnis dieser Koexistenz in der Perspektive von Komorbidität bzw. Multimorbidität verlangt konsequent auch die Identifikation gemeinsam geteilter pathogenetischer Mechanismen von emotionaler Dysregulation einerseits und neurologischen Krankheitsprozessen andererseits. Innerhalb eines biopsychosozialen Krankheitsmodells sind psychologische und psychosoziale Stressoren eigenständig zu erfassen, aber auch in ihren neurobiologischen Implikationen zu reflektieren. Vielfältige Einflüsse neurologischer Therapien auf das psychische Erleben wie auch umgekehrt psychiatrische und psychotherapeutische Interventionen auf neurologische Funktionen und Prozesse müssen beachtet werden.

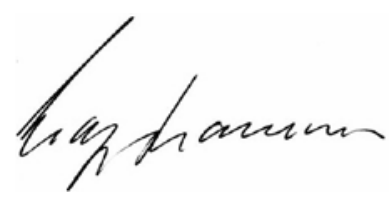

Prof. Dr. H.-P. Kapfhammer

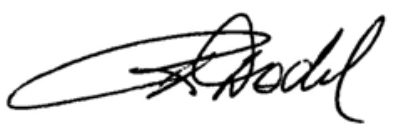

Prof. Dr. R.C. Dodel

\section{Korrespondenzadressen}

\section{Prof. Dr. Dr. H.-P- Kapfhammer}

Universitätsklinik für Psychiatrie,

Medizinische Universität Graz

Auenbruggerplatz 31, $8036 \mathrm{Graz}$

Österreich

hans-peter.kapfhammer@klinikum-graz.at

Prof. Dr. R. Dodel

Klinik und Poliklinik für Neurologie,

Philipps-Universität Marburg,

Universitätsklinikum Gießen und Marburg

Baldingerstr. 1, 35043 Marburg

dodel@med.uni-marburg.de
GENERATE - Deutsches Netzwerk zur Erforschung autoimmuner Enzephalitiden gegründet

Eine Gruppe von Neurologen aus ganz Deutschland gründete im Januar im Rahmen der ANIM in Hannover das Deutsche Netzwerk zur Erforschung autoimmuner Enzephalitiden. Der Name GENERATE steht für GErman NEtwork for Research on AuToimmune Encephalitis.

Übergeordnetes Ziel des Netzwerkes ist es, die Diagnostik, Therapie und Versorgung von Patienten mit autoimmunen Enzephalitiden zu verbessern. Das Netzwerk setzt sich zusammen aus einem nationalen Patientenregister als Basis klinischer Forschung und einzelnen wissenschaftlichen Arbeitsgruppen, die sich inhaltlich ergänzen.

Bei vielen autoimmunen Enzephalitiden können pathogene Autoantikörper nachgewiesen werden (z.B. gegen NMDAR, Lgl1, CASPR2, GAD, GABA(b)R, AMPAR, GlyR). Die anti-NMDAR Enzephalitis beispielsweise, stellt mittlerweile eine der häufigsten autoimmune Enzephalitiden neben der Neuromyelitis optica (NMO) dar. Zur NMO existiert bereits die eigenständige Neuromyelitis optica Studiengruppe (NEMOS, www.nemos-net.de) in Deutschland. Autoimmune antikörper-assoziierte Enzephalitiden sind gut behandelbar. Es bestehen jedoch bei behandelnden Ärzten Unsicherheiten zu sinnvoller Diagnostik, Behandlung und Prognose.

Die GENERATE Homepage bietet aktuelle Informationen zu Pathophysiologie, Diagnostik und Therapie autoimmuner Enzephalitiden. Patienten und Ärzte können dort spezialisierte Zentren finden, um rasch Informationen zur notwendigen Diagnostik und Behandlung zu erhalten.

Weitere Informationen: www.generate-net.de

Quelle: W. Köhler, Klinik für Neurologie und Neurologische Intensivmedizin Fachkrankenhaus Hubertusburg, Wermsdorf 